\title{
Cobertura con injerto dermoepidérmicos mediante técnica MEEK en el tratamiento de grandes quemados*
}

\author{
Drs. JOSÉ PIÑEROS B. ${ }^{1}$, RICARDO ROA G. ${ }^{1}$, ÁLVARO CUADRA C. ${ }^{1}$, CARLA MORA P. ${ }^{1}$, \\ KENNETH GULER G. ${ }^{1}$, NICOLÁS PEREIRA C. ${ }^{1}$, WILFREDO CALDERÓN O. ${ }^{1}$ \\ 1 Servicio de Cirugía Plástica y Quemados Hospital del Trabajador. \\ Santiago, Chile.
}

\section{Skin graft using MEEK technique for skin coverage in severe burned patients}

\section{Introducción}

El tratamiento de los grandes quemados, con quemaduras de $2^{\circ}$ grado profundo y $3^{\circ}$ grado, representan un desafío para el cirujano debido, entre otros factores, a la limitante que significa el déficit de zona dadora de autoinjertos ${ }^{1}$.

Con los avances realizados por las unidades de cuidados intensivos, la mortalidad en la primera fase del gran quemado ha disminuido considerablemente $^{2}$, sin embargo, el manejo quirúrgico de estos pacientes aún presenta limitaciones ${ }^{1}$. En relación a esto, se sabe que el pronóstico del paciente depende de la realización de escarectomía y cobertura pre$\mathrm{coz}^{3}$. Para restituir la cobertura en grandes quemados, a lo largo del tiempo se han desarrollado múltiples métodos de reparación, tales como autoinjertos, heteroinjertos, sustitutos cutáneos, y se maximiza la cobertura mediante expansión de injertos y uso de estampillas ${ }^{4}$. En el año 1963, se describió la técnica MEEK ${ }^{\circledR}$ que consiste en microinjertos expandidos en una gasa con el objetivo de lograr una distribución homogénea ${ }^{2}$, sin embargo, con el desarrollo de los injertos expandidos en 1964, esta técnica fue abandonada ${ }^{2}$.
Posteriormente, con el aumento de la sobrevida de los pacientes en fase inicial, los cirujanos de quemados se enfrentaron al desafío de coberturas más extensas, por lo que se generó nuevamente interés en optimizar la técnica mencionada, debido a que ofrece una expansión que puede llegar a 1:18 versus los injertos expandidos que sólo llegan a 1:6, limitando de este modo las zonas dadoras ${ }^{1}$.

A propósito de 4 casos de grandes quemados atendidos en el hospital en los últimos meses se presenta el protocolo de técnica MEEK ${ }^{\circledR}$ de nuestro servicio.

Inicialmente se realizan los aseos quirúrgicos y escarectomías requeridas, quedando cubiertos de manera transitoria con Biobrane.

Para realizar el injerto dermoepidérmico (IDE) se toman autoinjertos de 0,01 pulgadas de espesor con placas de 2 pulgadas de ancho, previa hidratación de la piel con solución fisiológica. Posteriormente se posicionan sobre corchos de $4,2 \mathrm{~cm}$ por $4,2 \mathrm{~cm}$, con la dermis hacia abajo y se recortan los excedentes de injerto (Figura 1). Este corcho, posteriormente fijado por enrejado metálico, se pasa por el carril del dermatomo que contiene 13 cuchillos paralelos espaciados por $3 \mathrm{~mm}$ (Figura 2), estos cortan los injertos

\footnotetext{
* Recibido el 12 de Abril de 2010 y aceptado para publicación el 14 de mayo de 2010.

Correspondencia: Dr. Wilfredo Calderón O. Ramón Carnicer $185,5^{\circ}$ piso, Santiago, Chile. E-mail: wcalderon@hts.cl
} 


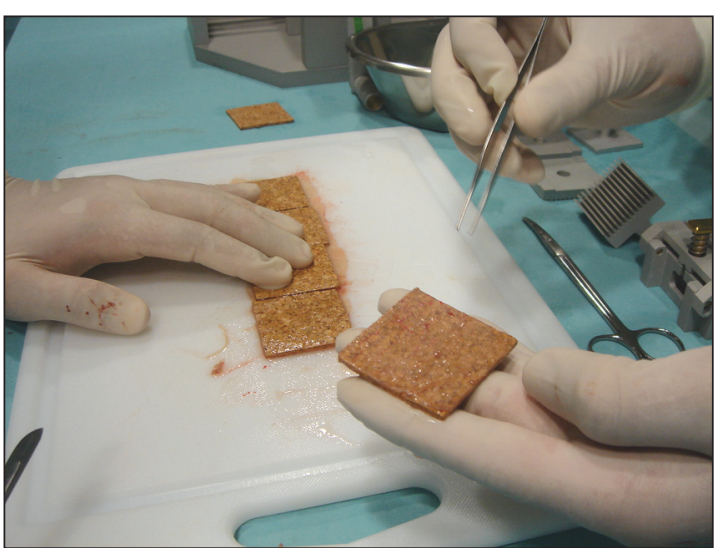

Figura 1. Corte de excedentes de IDE.

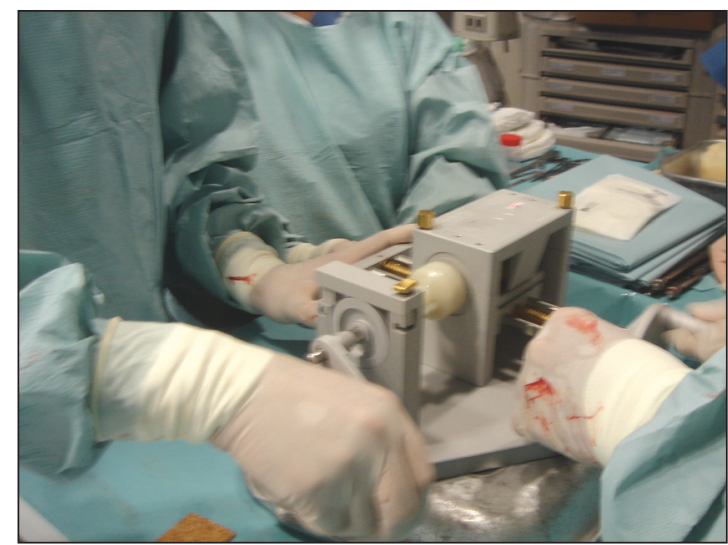

Figura 3. Paso por el dermátomo en sentido doble realizando cortes en cuadrícula.

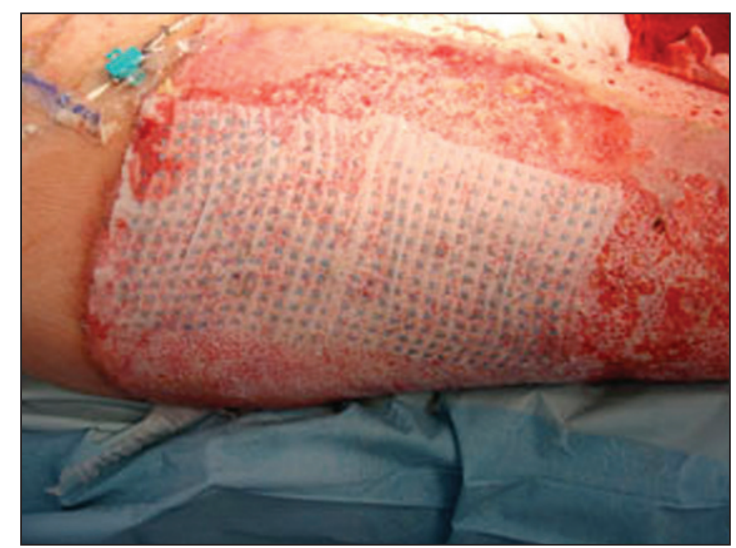

Figura 5. Injertos posicionados sobre zonas cruentas.

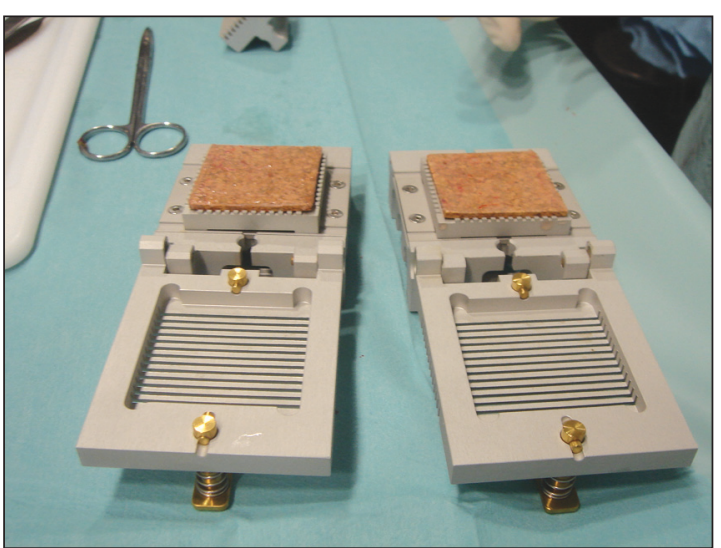

Figura 2. Colocación de injertos sobre corcho en el carrier.

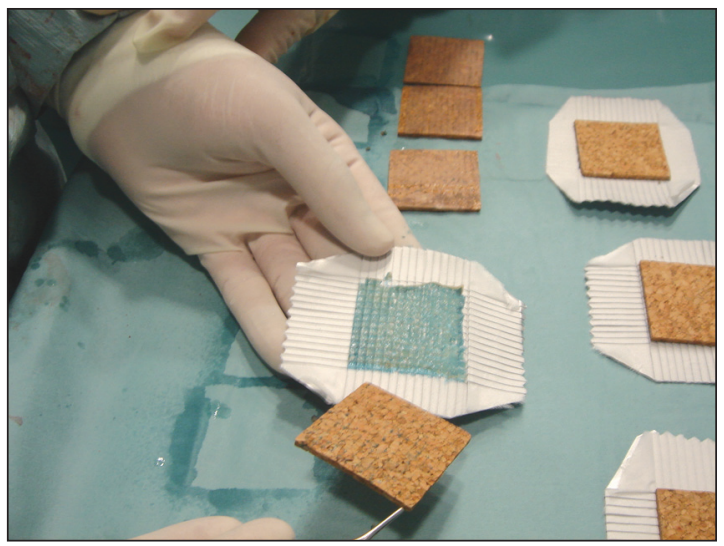

Figura 4. Injerto posicionado sobre papel metálico.

pero no los corchos. Posteriormente se rota el corcho en $90^{\circ}$ y se pasa nuevamente por el dermátomo, resultando 14 x 14 injertos de $3 \times 3$ mm (Figura 3). Se retira cuidadosamente el enrejado metálico y al injerto, aún posicionado sobre el corcho se le aplica un apósito adhesivo en spray. Se esperan dos a tres minutos y se adhiere a gasa plegada sobre placa de papel metálico (Figura 4), se retira este último y se expande gasa. Se posicionan injertos sobre gasa en zonas cruentas y se fijan mediante grapas ${ }^{5}$. Se realiza curación con Jelonet $\AA$, moltopren impregnado en Furacín ${ }^{\circledR}$ y apósitos secos. Las gasas se retiran al $7^{\circ}$ día en pabellón (Figura 5). 


\section{Casos}

\section{Caso 1}

Paciente de 54 años, fumadora, sufre quemaduras por fuego secundarias a explosión de gas. Resultando con quemaduras en un $70 \%$ de superficie corporal total, $2^{\circ}$ grado superficial y $2^{\circ}$ grado profunda en cara, tórax anterior y posterior, extremidades superiores e inferiores y lesión de vía aérea.

Ingreso con zonas cruentas en un $54 \%$, de $2^{\circ}$ grado, profunda y $3^{\circ}$ grado.

\section{Caso 2}

Paciente de 32 años sin antecedentes mórbidos. Mientras trabajaba en faena de carbón, enfriándolo en tambor con agua, se produce escape de agua a $140^{\circ} \mathrm{C}$ aproximadamente, resultando con quemaduras en un $54 \% \operatorname{SCT} 2^{\circ}$ grado, superficial y profundo y $3^{\circ}$ grado en cara, cuello, tórax anterior, extremidades superiores y extremidades inferiores, e injuria inhalatoria.

\section{Caso 3}

Paciente de 46 años sin antecedentes mórbidos. Mientras trabajaba cerca de tablero eléctrico sufre quemadura por arco eléctrico resultando con lesiones en un 70\% superficie corporal total (SCT); 7\% SCT $2^{\circ}$ grado profundo en cabeza; $2 \%$ SCT $2^{\circ}$ grado profundo cervical anterior y posterior; $2^{\circ}$ grado profundo $36 \%$ SCT tórax anterior, posterior y abdomen; $9 \%$ SCT $2^{\circ}$ grado profundo EESS; $3 \%$ SCT $1^{\circ}$ grado extremidad inferior derecha, más injuria inhalatoria.

\section{Caso 4}

Paciente de 50 años sin antecedentes mórbidos, que cae en piscina termal a $80^{\circ} \mathrm{C}$ resultando con quemaduras $2^{\circ}$ grado superficial y profunda, $69 \%$ SCT en tronco y extremidades, sin lesiones asociadas.

Se realizan múltiples aseos quirúrgicos y escarectomías, quedando cubierto con Biobrane ${ }^{\circledR}$, previo a su cobertura definitiva.

\section{Resultados}

Los resultados se presentan en la Tabla 1.

El primer caso requirió IDE en un $15 \%$ con MEEK en un tiempo quirúrgico, lográndose un prendimiento de un $95 \%$ y una epitelización entre microinjertos de un $100 \%$ a las 4 semanas.

El segundo caso requirió injerto con técnica MEEK $®$ en un $45 \%$ en extremidad superior izquierda y ambas extremidades inferiores, en 3 tiempos quirúrgicos, con un prendimiento de un $100 \%$ y una epitelización entre microinjertos a las 4 semanas de un $100 \%$ (Figura 6).

El tercer caso requirió IDE con técnica MEEK ${ }^{\circledR}$ en un $36 \%$ en ambas extremidades superiores, cara anterior del tórax y abdomen, en 3 tiempos quirúrgicos, con un prendimiento de un $100 \%$ y epitelización entre microinjertos a las 4 semanas de un $100 \%$ (Figura 7).

El cuarto caso requirió IDE MEEK ${ }^{\circledR}$ en un $45 \%$ en abdomen, extremidad superior derecha y ambas extremidades inferiores, en 4 tiempos quirúrgicos, con un prendimiento de un $30 \%$ a un $100 \%$ en las distintas cirugías, y una epitelización de un $100 \%$ a las 4 semanas.

A contar desde el ingreso, la cobertura definitiva se inició entre la $1^{\circ}$ y $4^{\circ}$ semana, realizándose el total de la cobertura en un máximo de 5 semanas. El promedio de superficie injertada fue de un $35 \%$, con un prendimiento de un $91 \%$ y epitelización total a las 4 semanas de un $100 \%$.

Tabla 1. Resultados

\begin{tabular}{lcccccccccc}
\hline Paciente & Edad & Sexo & $\begin{array}{c}\text { Antecedentes } \\
\text { mórbidos }\end{array}$ & SCQ* & IG** & Agente & $\begin{array}{c}\text { Superficie } \\
\text { Injertada }\end{array}$ & $\begin{array}{c}\text { Prendi- } \\
\text { miento }\end{array}$ & $\begin{array}{c}\text { Epiteliza- } \\
\text { ción a las 4 } \\
\text { semanas }\end{array}$ & $\begin{array}{c}\text { Lesiones } \\
\text { asociadas }\end{array}$ \\
\hline Caso 1 & $54 \mathrm{a}$ & F & Tabaquismo & $70 \%$ & 234 & Fuego & $15 \%$ & $95 \%$ & $100 \%$ & Inhalatoria \\
Caso 2 & $32 \mathrm{a}$ & M & No & $54 \%$ & 200 & Agua & $45 \%$ & $100 \%$ & $100 \%$ & Inhalatoria \\
Caso 3 & $46 \mathrm{a}$ & M & No & $59 \%$ & 177 & Fuego & $31 \%$ & $100 \%$ & $100 \%$ & Inhalatoria \\
Caso 4 & 54 a & M & No & $69 \%$ & 188 & Agua & $49 \%$ & $70 \%$ & $100 \%$ & No \\
\hline
\end{tabular}

*Superficie corporal quemada. **índice de gravedad. (Según fórmula de Garcés: Edad + $(\% \mathrm{~A}$ x1) + $(\% \mathrm{ABx} 2)+(\% \mathrm{Bx} 3)+$ (20 puntos en caso de patología agregada, trauma asociado, injuria inhalatoria, quemadura eléctrica) $+(10$ puntos por malas condiciones socioeconómicas). 


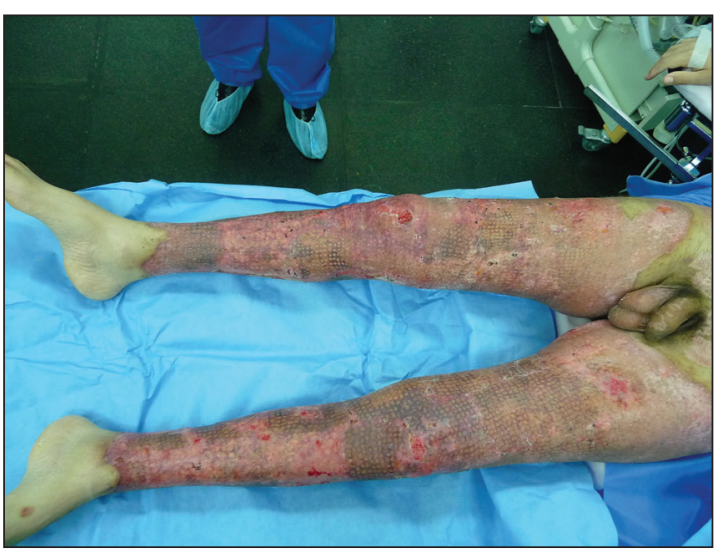

Figura 6. Extremidades inferiores epidermizadas.

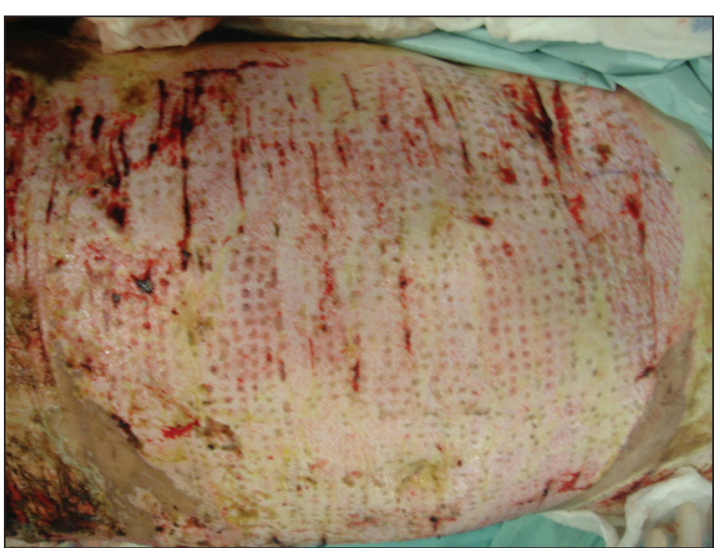

Figura 7. Tronco y abdomen injertado, en vías de epitelización.

\section{Referencias}

1. Kreis RW, Mackie DP, Vloemans AW, Hermans RP, Hoekstra MJ. Widely expanded postage stamp skin grafts using a modified Meek technique in combination with an allograft overlay. Burns 1993; 19: 142-145.

2. Lari AR, Gang RK. Expansion technique for skin grafts (MEEK technique) in the treatment of severely burned patients. Burns 2001; 27: 61-66.

3. Atiyeh BS, Hayek SN, Gunn SW. New technologies for burn wound closure and healing--review of the literature. Burns 2005; 8: 944-956.

4. Lee SS, Tsai CC, Lai CS, Lin SD. An easy method for preparation of postage stamp autografts. Burns 2000; 26: 741-749.

5. Lee SS, Tsai CC, Lai CS, Lin SD. An easy method for preparation of postage stamp autografts. Burns 2000; 26: 741-749.

6. Zermani RG, Zarabini A, Trivisonno A. Micrografting in the treatment of severely burned patients. Burns 1997;23: 604-607.

7. Hsieh CS, Schuong JY, Huang WS, Huang TT. Five years' experience of the modified Meek technique in the management of extensive burns. Burns 2008; 34: 350-354.

8. Still JM Jr, Orlet HK, Law EJ. Use of cultured epidermal autografts in the treatment of large burns. Burns 1994;20 : 539-541

9. Lee SS, Lin TM, Chen YH, Lin SD, Lai CS. "Flypaper technique" a modified expansion method for preparation of postage stamp autografts. Burns 2005; 31: 753-757.

10. Lee SS, Chen YH, Sun IF, Chen MC, Lin SD, Lai CS. "Shift to right flypaper technique" a refined method for postage stamp autografting preparation. Burns 2007; 33 : 764-769. 